\title{
Analysis of nonlinear structural dynamics and resonance in trees
}

\author{
H. Doumiri Ganji ${ }^{\mathrm{a}, *}$, S.S. Ganji ${ }^{\mathrm{a}, *}$, D.D. Ganji ${ }^{\mathrm{b}}$ and F. Vaseghi ${ }^{\mathrm{c}}$ \\ ${ }^{a}$ Young Researchers Club, Science and Research Branch, Islamic Azad University, Tehran, Iran \\ ${ }^{\mathrm{b}}$ Department of Mechanical Engineering, Babol University of Technology, Babol, Iran \\ ${ }^{\mathrm{c}}$ Department of Industerial Engineering, Iran University of Science and Technology, Tehran, Iran
}

Received 23 January 2011

Revised 17 August 2011

\begin{abstract}
Wind and gravity both impact trees in storms, but wind loads greatly exceed gravity loads in most situations. Complex behavior of trees in windstorms is gradually turning into a controversial concern among ecological engineers. To better understand the effects of nonlinear behavior of trees, the dynamic forces on tree structures during periods of high winds have been examined as a mass-spring system. In fact, the simulated dynamic forces created by strong winds are studied in order to determine the responses of the trees to such dynamic loads. Many of such nonlinear differential equations are complicated to solve. Therefore, this paper focuses on an accurate and simple solution, Differential Transformation Method (DTM), to solve the derived equation. In this regard, the concept of differential transformation is briefly introduced. The approximate solution to this equation is calculated in the form of a series with easily computable terms. Then, the method has been employed to achieve an acceptable solution to the presented nonlinear differential equation. To verify the accuracy of the proposed method, the obtained results from DTM are compared with those from the numerical solution. The results reveal that this method gives successive approximations of high accuracy solution.
\end{abstract}

Keywords: Tree, mass-spring system, oscillation, runge-kutta method, analytical solution

\section{Introduction}

Wind damage has always been a global phenomenon that affects tropical, temperate and plantation forests [1], such as Hurricane Hugo in South Carolina [2] and Storm Vivian in Northern Europe [3]. Such storms severely affect forest management. Wind loads are the largest dynamic loads on trees [4]. Loads caused by wind are periodic and create a sway motion in trees which can be simplified using a conceptual model of a tree stem [5] without branches and being considered as an upside-down pendulum [6]. Recently, there have been a variety of models considering resonance behavior in plant stems [7]. A number of mathematical models such as mass-spring-damper systems have been proposed to anticipate stem dynamics and natural frequencies [8].

In most cases, such problems do not admit analytical solution, so these equations have to be solved using special techniques. In recent years, much attention has been devoted to the newly developed methods to construct an analytic solution to these equations, including the Perturbation techniques. Perturbation techniques are too strongly dependent upon the so-called "small parameters" [9]. Many other methods have been introduced to solve nonlinear equations such as homotopy perturbation [10-13] and analysis methods [14,15], variational approach [16,17], parameter expanding [18,28], Max-Min Approach [MMA] [19], and Amplitude-Frequency Formulation (AFF) [20, 27]. One of the semi-exact methods which do not need linearization is differential transform method (DTM) which has attracted many authors to use this method for solving nonlinear oscillator dynamic problems [21-23].

${ }^{*}$ Corresponding authors. E-mail: r.alizadehganji@gmail.com; hd.ganji@gmail.com. 


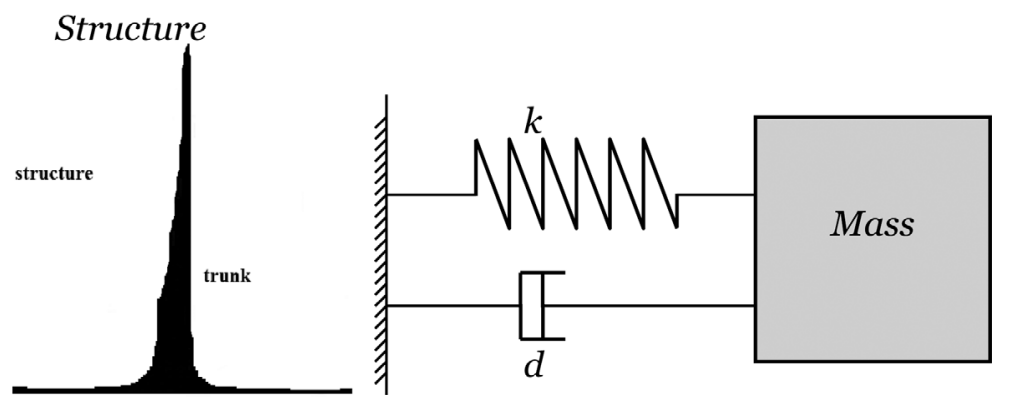

Fig. 1. Dynamic model of a tree trunk (structure) represented as a mass $(m)$ oscillating on a spring $(k)$ with the motion being damped $(d)$.
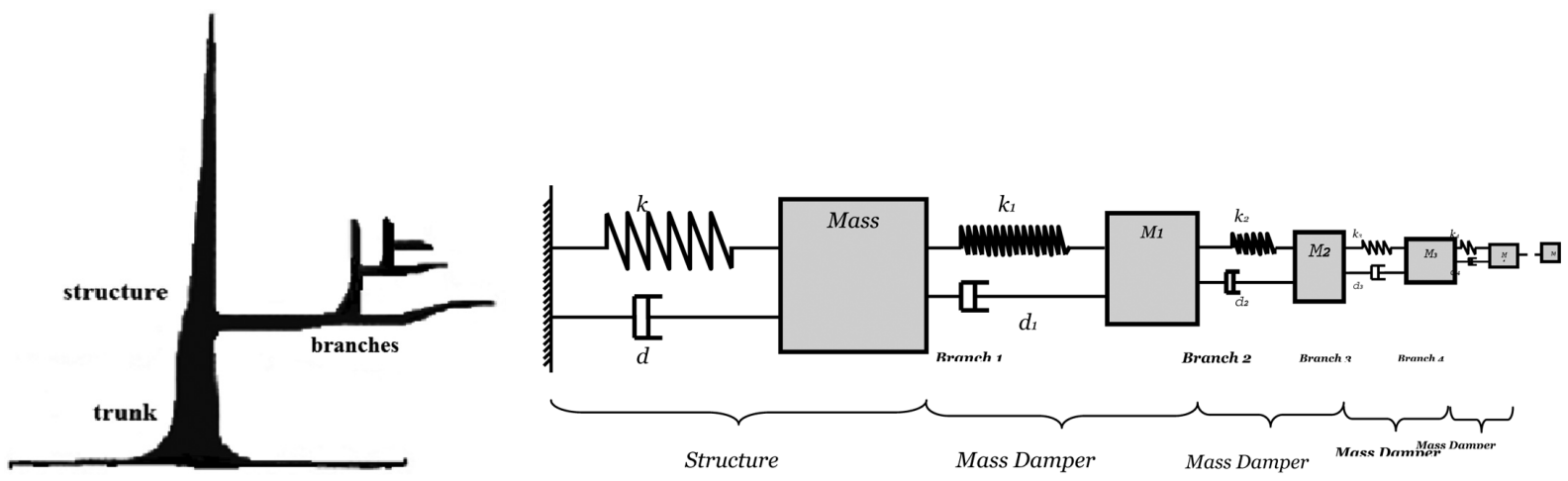

Fig. 2. Complex branching of a tree and equivalent mass-damping model.

The paper is organized as following. Section 2 outlines the nonlinear second-order differential equation derived from Mass-Spring-System [24]. Section 3 describes Differential Transformation Method. Section 4 applies the presented approximate method, DTM, to solve the derived Duffing equation. Results and discussions are presented in Section 5. Finally, further findings are discussed in conclusions.

\section{Problem statement and mathematical formulation}

A tree trunk in storm can be simulated as a mass $(\mathrm{m})$ oscillating on a hooking spring of negligible mass $(k)$ and has some damping $(d)$, Fig. 1 [6]. The second order branches are equivalent to adding a mass damper to the first mass damper. In the other word, the first-order branches are themselves mass damped with second order branches. The smaller branches also affect on the second-order branch Fig. 2. The effect of the branch on the tree trunk is illustrated in Fig. 3. The branch $(m)$ oscillates on a spring $\left(k_{1}\right)$, and the motion is damped $\left(d_{1}\right)$ due to internal damping.

The simplest model, plant stem structural dynamics and resonance, is a simple spring-mass-damper system as the following linear ordinary differential equation (ODE) [8].

$$
m x^{\prime \prime}(t)+c x^{\prime}(t)+k_{1} x(t)=F(t),
$$

Where $m, c, k_{1}$ are the mass, damping coefficient, and proportional to the stiffness of the spring respectively. In adition, $F(t)$ is the applied force as a function of time and $x(t)$ gives the displacement of the stem as a function of time. The Duffing oscillator was originally introduced in relation to the single (spatial) modes of vibration of a steel beam subjected to external periodic forces. Miller has modeled tree as a Duffing oscillator, and after adding a cubic stiffness term, $k^{3} x^{3}(t)$ to the left-hand side of Eq. (1), and doing some assumptions and calculations, the Eq. (2) has been derived [26]:

$$
X^{\prime \prime}(T)+2 \zeta X^{\prime}(T)+X(T)+\frac{K_{3}}{K_{1}} X^{3}(T)=P F(\Omega T),
$$



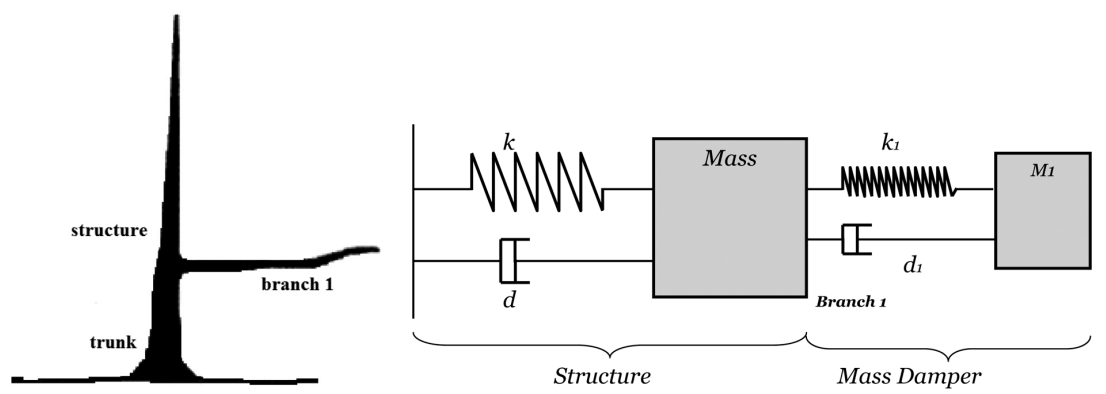

Fig. 3. Dynamic model of a trunk with branch (mass damper) attached.

$$
K_{1}=k_{1} L_{\max }, K_{3}=k_{3}\left(L_{\max }\right)^{3}, P=\frac{p_{0}}{K_{1}}, \quad \Omega=\frac{\omega}{\omega_{0}}, \quad X=\frac{x}{L_{\max }}, T=\omega_{0} t, .
$$

Where $k_{3}$ is the stiffness coefficient. $L_{\max }$ is a characteristic maximum deflection. In this case, $L_{\max }$ is the deflection at which failure occurs with well known oscillator boundary conditions as follows

$$
X(0)=1, \quad X^{\prime}(0)=0 .
$$

Where $K_{3} / K_{1}$ and $p_{0} / K_{1}$ will be referred to as the nonlinear ratio and the normalized forcing amplitude, respectively. The forcing term could be set to the following:

$$
F(\Omega T)=\frac{1}{2}(1+\sin (\Omega T)) \text {. }
$$

\section{Fundamentals of differential transformation method}

We suppose $x(t)$ to be analytic function in a domain $D$ and $t=t_{i}$ represent any point in $D$. The function $x(t)$ is then represented by one power series whose center is located at $t_{i}$. The Taylor series expansion function of $x(t)$ is of the form $[25,26]$.

$$
x(t)=\sum_{k=0}^{\infty} \frac{\left(t-t_{i}\right)^{k}}{k !}\left[\frac{d^{k} x(t)}{d t^{k}}\right]_{t=t_{i}} \quad \forall t \in D,
$$

When $t_{i}=0$ is referred to as the Maclaurin series of $x(t)$ and is expressed as:

$$
x(t)=\sum_{k=0}^{\infty} \frac{t^{k}}{k !}\left[\frac{d^{k} x(t)}{d t^{k}}\right]_{t=0} \quad \forall t \in D,
$$

The differential transformation of the function $x(t)$ is defined as follows:

$$
X(k)=\sum_{k=0}^{\infty} \frac{H^{k}}{k !}\left[\frac{d^{k} x(t)}{d t^{k}}\right]_{t=0},
$$

Where $x(t)$ the original is function and $X(k)$ is the transformed function. The differential spectrum of $X(k)$ is confined within the interval $t \in[0, H]$, where $H$ is a constant. The differential inverse transform of $X(k)$ is defined as follows:

$$
x(t)=\sum_{k=0}^{\infty}\left(\frac{t}{H}\right)^{k} X(k)
$$

It is clear that the concept of differential transformation is based upon the Taylor series expansion. The values of function $X(k)$ at $\mathrm{t}$ values of argument $k$ are referred to as discrete, i.e. $X(0)$ is known as the zero discrete, $X(1)$ as the first discrete, etc. The more discrete available, the more precisely it is possible to restore the unknown function. The function $x(t)$ consists of the T-function $X(k)$, and its value is given by the sum of the T-function with $(t / H)^{k}$ 
Table 1

The fundamental operations of differential transform method

\begin{tabular}{ll}
\hline Original function & Transformed function \\
\hline$x(t)=\alpha f(x) \pm \beta g(t)$ & $X(k)=\alpha F(k) \pm \beta G(k)$ \\
$x(t)=\frac{d f(t)}{d t}$ & $X(k)=(k+1) F(k+1)$ \\
$x(t)=\frac{d^{2} f(t)}{d t^{2}}$ & $X(k)=(k+1)(k+2) F(k+2)$ \\
$x(t)=\exp (t)$ & $X(k)=\frac{k}{k !}$ \\
$x(t)=f(t) g(t)$ & $X(k)=\sum_{l=0}^{k} F(l) G(k-l)$ \\
$x(t)=t^{m}$ & $X(k)=\delta(k-m)= \begin{cases}1 & k=m \\
0 & k \neq m\end{cases}$ \\
$x(t)=\sin (\omega t+\alpha)$ & $X(k)=\frac{\omega^{k}}{k !} \sin \left(\frac{\pi k}{2}+\alpha\right)$ \\
$x(t)=\cos (\omega t+\alpha)$ & $X(k)=\frac{\omega^{k}}{k !} \cos \left(\frac{\pi k}{2}+\alpha\right)$ \\
\hline
\end{tabular}

Table 2

The comparison between the Numerical and DTM solution of $X(T)$ for various $P$, when $\zeta=0.1, \Omega=0.5$ and $\frac{K_{3}}{K_{1}}=1$

\begin{tabular}{|c|c|c|c|c|c|c|}
\hline \multirow[b]{2}{*}{$\mathrm{T}$} & \multicolumn{2}{|c|}{$P=0.25$} & \multicolumn{2}{|c|}{$P=0.33$} & \multicolumn{2}{|c|}{$P=0.66$} \\
\hline & DTM & $\mathrm{NM}$ & DTM & $\mathrm{NM}$ & DTM & NM \\
\hline 0.0 & 1.00000000 & 1.00000000 & 1.00000000 & 1.00000000 & 1.00000000 & 1.00000000 \\
\hline 0.1 & 0.99072841 & 0.99072841 & 0.99092973 & 0.99092973 & 0.99176020 & 0.99176020 \\
\hline 0.2 & 0.96356097 & 0.96356098 & 0.96436638 & 0.96436639 & 0.96768863 & 0.96768864 \\
\hline 0.3 & 0.91994303 & 0.91994304 & 0.92174499 & 0.92174500 & 0.92917752 & 0.92917753 \\
\hline 0.4 & 0.86182862 & 0.86182863 & 0.86499891 & 0.86499892 & 0.87807321 & 0.87807323 \\
\hline 0.5 & 0.79149639 & 0.79149641 & 0.79638100 & 0.79638102 & 0.81651873 & 0.81651875 \\
\hline 0.6 & 0.71135966 & 0.71135969 & 0.71827917 & 0.71827920 & 0.74679094 & 0.74679097 \\
\hline 0.7 & 0.62380022 & 0.62380028 & 0.63305442 & 0.63305448 & 0.67115607 & 0.67115613 \\
\hline 0.8 & 0.53104455 & 0.53104464 & 0.54291946 & 0.54291955 & 0.59175860 & 0.59175867 \\
\hline 0.9 & 0.43508893 & 0.43508900 & 0.44986413 & 0.44986419 & 0.51054920 & 0.51054923 \\
\hline 1.0 & 0.33767031 & 0.33767034 & 0.35562469 & 0.35562471 & 0.42924955 & 0.42924957 \\
\hline
\end{tabular}

as its coefficient. In real applications, at the right choice of constant $H$, the larger values of argument $k$ the discrete of spectrum reduce rapidly. The function $x(t)$ is expressed by a finite series and Eq. (8) can be written as:

$$
x(t)=\sum_{k=0}^{n}\left(\frac{t}{H}\right)^{k} X(k) .
$$

Mathematical operations performed by differential transform method are listed in Table 1.

\section{Solution with differential transformation method}

Equation (3) can be expressed as two simultaneous first-order differential equations written in terms of $X(T)$ and $Y(T)$, i.e.

Let

$$
Y(T)=X^{\prime}(T)
$$

Substituting Eq. (10) into Eqs (2) and (3) yields

$$
Y^{\prime}(T)+2 \zeta Y(T)+X(T)+\frac{K_{3}}{K_{1}} X^{3}(T)-F(\Omega T)=\frac{P}{2}(1+\sin (\Omega T)),
$$

And the initial conditions become

$$
X(0)=1, \quad Y(0)=0
$$

By a process of inverse differential transformation, the solutions of each sub-domain take $m+1$ terms for the power series, 
Table 3

The comparison between the Numerical and DTM solution of $X^{\prime}(T)$ for various $P$ when $\zeta=0.1, \Omega=0.5$ and $\frac{K_{3}}{K_{1}}=1$

\begin{tabular}{|c|c|c|c|c|c|c|}
\hline \multirow[b]{2}{*}{$\mathrm{T}$} & \multicolumn{2}{|c|}{$P=0.25$} & \multicolumn{2}{|c|}{$P=0.33$} & \multicolumn{2}{|c|}{$P=0.66$} \\
\hline & DTM & NM & DTM & NM & DTM & NM \\
\hline 0.0 & 0.00000000 & 0.00000000 & 0.00000000 & 0.00000000 & 0.00000000 & 0.00000000 \\
\hline 0.1 & -0.18409815 & -0.18409818 & -0.18006503 & -0.18006506 & -0.16342845 & -0.16342848 \\
\hline 0.2 & -0.35681688 & -0.35681689 & -0.34878620 & -0.34878621 & -0.31566123 & -0.31566124 \\
\hline 0.3 & -0.51225376 & -0.51225378 & -0.50038870 & -0.50038872 & -0.45145685 & -0.45145687 \\
\hline 0.4 & -0.64620423 & -0.64620425 & -0.63074669 & -0.63074671 & -0.56702983 & -0.56702986 \\
\hline 0.5 & -0.75638996 & -0.75638994 & -0.73760425 & -0.73760424 & -0.66024063 & -0.66024065 \\
\hline 0.6 & -0.84235428 & -0.84235426 & -0.82047707 & -0.82047705 & -0.73051946 & -0.73051949 \\
\hline 0.7 & -0.90511135 & -0.90511138 & -0.88031768 & -0.88031772 & -0.77859181 & -0.77859189 \\
\hline 0.8 & -0.94667031 & -0.94667038 & -0.91906018 & -0.91906026 & -0.80610085 & -0.80610094 \\
\hline 0.9 & -0.96954856 & -0.96954856 & -0.93915366 & -0.93915366 & -0.81521703 & -0.81521702 \\
\hline 1.0 & -0.97635478 & -0.97635464 & -0.94316194 & -0.94316181 & -0.80830013 & -0.80830003 \\
\hline
\end{tabular}

Table 4

The comparison between the Numerical and DTM solution of $X(T)$ for various $\frac{K_{3}}{K_{1}}$ when $\zeta=0.1, \Omega=0.5$ and $P=1$

\begin{tabular}{|c|c|c|c|c|c|c|}
\hline \multirow[b]{2}{*}{$\mathrm{T}$} & \multicolumn{2}{|c|}{$\frac{K_{3}}{K_{1}}=-0.5$} & \multicolumn{2}{|c|}{$\frac{K_{3}}{K_{1}}=1$} & \multicolumn{2}{|c|}{$\frac{K_{3}}{K_{1}}=2$} \\
\hline & DTM & NM & DTM & NM & DTM & NM \\
\hline 0.0 & 1.00000000 & 1.00000000 & 1.00000000 & 1.00000000 & 1.00000000 & 1.00000000 \\
\hline 0.1 & 1.00004147 & 1.00004146 & 0.99261584 & 0.99261584 & 0.98769609 & 0.98769609 \\
\hline 0.2 & 1.00033019 & 1.00033022 & 0.97111146 & 0.97111147 & 0.95210428 & 0.95210429 \\
\hline 0.3 & 1.00110956 & 1.00110957 & 0.93683428 & 0.93683429 & 0.89623789 & 0.89623790 \\
\hline 0.4 & 1.00261934 & 1.00261937 & 0.89153837 & 0.89153839 & 0.82408733 & 0.82408736 \\
\hline 0.5 & 1.00509641 & 1.00509642 & 0.83724753 & 0.83724755 & 0.74006451 & 0.74006456 \\
\hline 0.6 & 1.00877548 & 1.00877551 & 0.77611353 & 0.77611356 & 0.64850332 & 0.64850339 \\
\hline 0.7 & 1.01389001 & 1.01389003 & 0.71028909 & 0.71028914 & 0.55330700 & 0.55330708 \\
\hline 0.8 & 1.02067329 & 1.02067331 & 0.64182798 & 0.64182803 & 0.45776627 & 0.45776637 \\
\hline 0.9 & 1.02935981 & 1.02935982 & 0.57261710 & 0.57261711 & 0.36452237 & 0.36452246 \\
\hline 1.0 & 1.04018712 & 1.04018713 & 0.50433923 & 0.50433924 & 0.27562307 & 0.27562626 \\
\hline
\end{tabular}

Table 5

The comparison between the Numerical and DTM solution of $X^{\prime}(T)$ for various $\frac{K_{3}}{K_{1}}$ when $\zeta=0.1, \Omega=0.5$ and $P=1$

\begin{tabular}{|c|c|c|c|c|c|c|}
\hline \multirow[b]{2}{*}{$\mathrm{T}$} & \multicolumn{2}{|c|}{$\frac{K_{3}}{K_{1}}=-0.5$} & \multicolumn{2}{|c|}{$\frac{K_{3}}{K_{1}}=1$} & \multicolumn{2}{|c|}{$\frac{K_{3}}{K_{1}}=2$} \\
\hline & DTM & NM & DTM & NM & DTM & NM \\
\hline 0.0 & 0.00000000 & 0.00000000 & 0.00000000 & 0.00000000 & 0.00000000 & 0.00000000 \\
\hline 0.1 & 0.00124197 & 0.00124197 & -0.14628782 & -0.14628784 & -0.24342291 & -0.24342291 \\
\hline 0.2 & 0.00493807 & 0.00493807 & -0.28153515 & -0.28153517 & -0.46330940 & -0.46330943 \\
\hline 0.3 & 0.01104881 & 0.01104878 & -0.40106172 & -0.40106175 & -0.64727139 & -0.64727141 \\
\hline 0.4 & 0.01954155 & 0.01954154 & -0.50145932 & -0.50145935 & -0.78830116 & -0.78830117 \\
\hline 0.5 & 0.03039087 & 0.03039082 & -0.58074974 & -0.58074978 & -0.88487294 & -0.88487302 \\
\hline 0.6 & 0.04357947 & 0.04357946 & -0.63832564 & -0.63832571 & -0.93981588 & -0.93981598 \\
\hline 0.7 & 0.05909973 & 0.05909967 & -0.67472888 & -0.67472898 & -0.95862114 & -0.95862123 \\
\hline 0.8 & 0.07695631 & 0.07695629 & -0.69134246 & -0.69134255 & -0.94780251 & -0.94780259 \\
\hline 0.9 & 0.09716965 & 0.09716959 & -0.69006859 & -0.69006856 & -0.91367591 & -0.91367561 \\
\hline 1.0 & 0.11978098 & 0.11978091 & -0.67304615 & -0.67304611 & -0.86185569 & -0.86165376 \\
\hline
\end{tabular}

$$
\begin{aligned}
& X_{i}(T)=\sum_{k=0}^{m}\left(\frac{T}{H_{i}}\right)^{k} A_{i}(k), 0 \leqslant T \leqslant H_{i}, \\
& Y_{i}(T)=\sum_{k=0}^{m}\left(\frac{T}{H_{i}}\right)^{k} B_{i}(k), 0 \leqslant T \leqslant H_{i},
\end{aligned}
$$

Where $i=0,1,2, \ldots, n$ illustrates the $\mathrm{i}^{t h}$ sub-domain, $k=0,1,2, \ldots, m$ shows the term of the power series, $H_{i}$ also shows the sub-domain interval, and $A_{i}(k)$ and $B_{i}(k)$ are the transformed functions of $X_{i}(T)$ and $Y_{i}(T)$, 
Table 6

The comparison between the Numerical and DTM solution of $X(1)$ and $X^{\prime}(1)$ for various $\Omega$ when $\zeta=0.1, \frac{K_{3}}{K_{1}}=2$ and $P=1.5$

\begin{tabular}{cccccc}
\hline & \multicolumn{2}{c}{$X(1)$} & & \multicolumn{2}{c}{$X^{\prime}(1)$} \\
\cline { 2 - 3 } \cline { 5 - 6 }$\Omega$ & DTM & NM & & DTM & NM \\
\hline 0.1 & 0.3380846670 & 0.3380853546 & & -0.7870958690 & -0.7870497125 \\
0.5 & 0.3776158092 & 0.3776163128 & & -0.6775060731 & -0.6774719398 \\
1.0 & 0.4228342293 & 0.4228342750 & & -0.5612676581 & -0.5612619449 \\
1.5 & 0.4601121634 & 0.4601115543 & & -0.4828478278 & -0.4828818811 \\
2.0 & 0.4868695578 & 0.4868682977 & & -0.4517814955 & -0.4518550469 \\
\hline
\end{tabular}

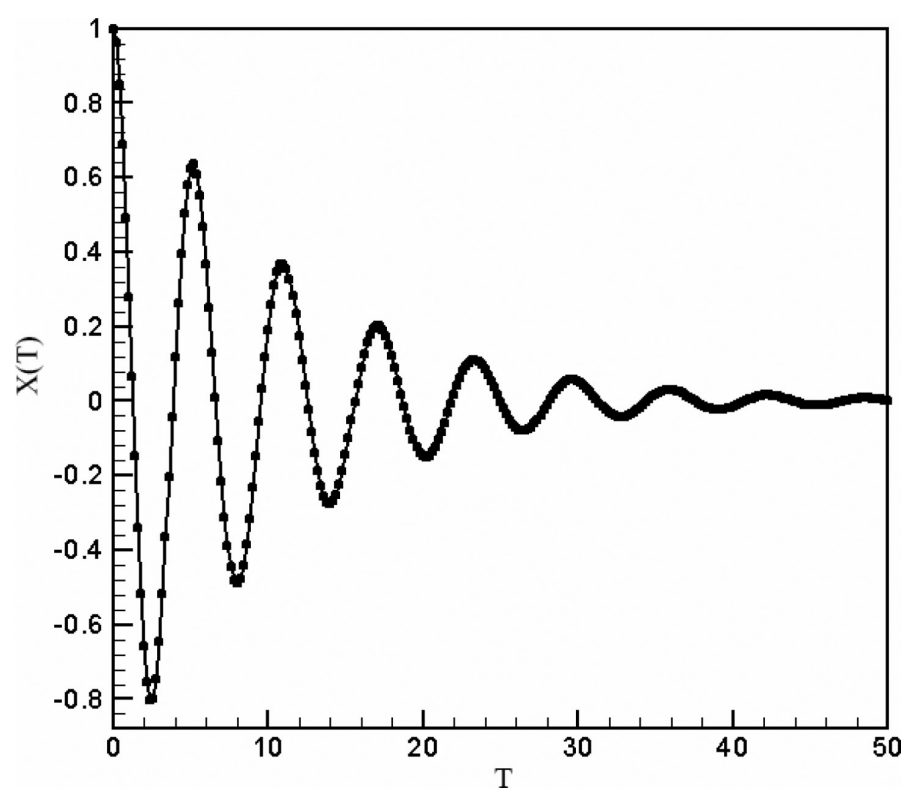

Fig. 4. The comparison between the numerical solution and DTM solution of $X(T)$ when $\zeta=0.1, \frac{K_{3}}{K_{1}}=1, \Omega=0.75$ and $P=0$ which solid line denote DTM solution and symbols show the numeric solution.

respectively. As the initial conditions Eq. (23),

$$
\begin{aligned}
& A_{0}(0)= \begin{cases}1 & k=0 \\
0 & k \neq 0\end{cases} \\
& B_{0}(0)=0 .
\end{aligned}
$$

The differential transformation Eqs (10) and (11) yields the following

$$
\begin{aligned}
& \frac{k+1}{H_{i}} A_{i}(k+1)=B_{i}(k), \\
& \frac{k+1}{H_{i}} B_{i}(k+1)+2 \zeta B_{i}(k)+A_{i}(k)+\frac{K_{3}}{K_{1}} \sum_{l=0}^{k} A_{k-l} \sum_{m=0}^{l} A_{l-m} A_{m}=\frac{P}{2}\left(1+\frac{\Omega^{k}}{k !} \sin \left(\frac{\pi k}{2}\right)\right),
\end{aligned}
$$

It can be clearly seen in Tables 2-5, there are acceptable results, which confirms the validity of the DTM. As far as Tables 2 and 3 are concerned, the comparisons are shown between the numerical and DTM solutions in terms of $X(T)$ and $X^{\prime}(T)$ regarding various normalized forcing amplitudes, $P \quad \zeta=0.1, \Omega=0.5$ and $K_{3} / K_{1}=1$. Moreover, Tables 4 and 5 show the high accuracy of this method in comparison with RK4 concerning $\zeta=0.1$, $\Omega=0.5, P=1$ as well as various nonlinear ratio, $K_{3} / K_{1}$. In this view, Tables 6 and 7 indicate the accuracy of 
Table 7

The comparison between the Numerical and DTM solution of $X(1)$ and $X^{\prime}(1)$ for various $\zeta$ when $\Omega=1.5, \frac{K_{3}}{K_{1}}=2$ and $P=1.5$

\begin{tabular}{cccccc}
\hline & \multicolumn{2}{c}{$X(1)$} & & \multicolumn{2}{c}{$X^{\prime}(1)$} \\
\cline { 2 - 3 } \cline { 5 - 6 }$\zeta$ & DTM & NM & & DTM & NM \\
\hline 0.1 & 0.4601121639 & 0.4601115543 & & -0.4828478272 & -0.4828818811 \\
0.2 & 0.4931828855 & 0.4931818360 & & -0.4224228100 & -0.4224887277 \\
0.3 & 0.5228903779 & 0.5228898523 & & -0.3720973110 & -0.3721237376 \\
0.5 & 0.5738232512 & 0.5738245146 & & -0.2949923900 & -0.2949073666 \\
1.0 & 0.6653998852 & 0.6654019226 & & -0.1877939544 & -0.1876672343 \\
\hline
\end{tabular}

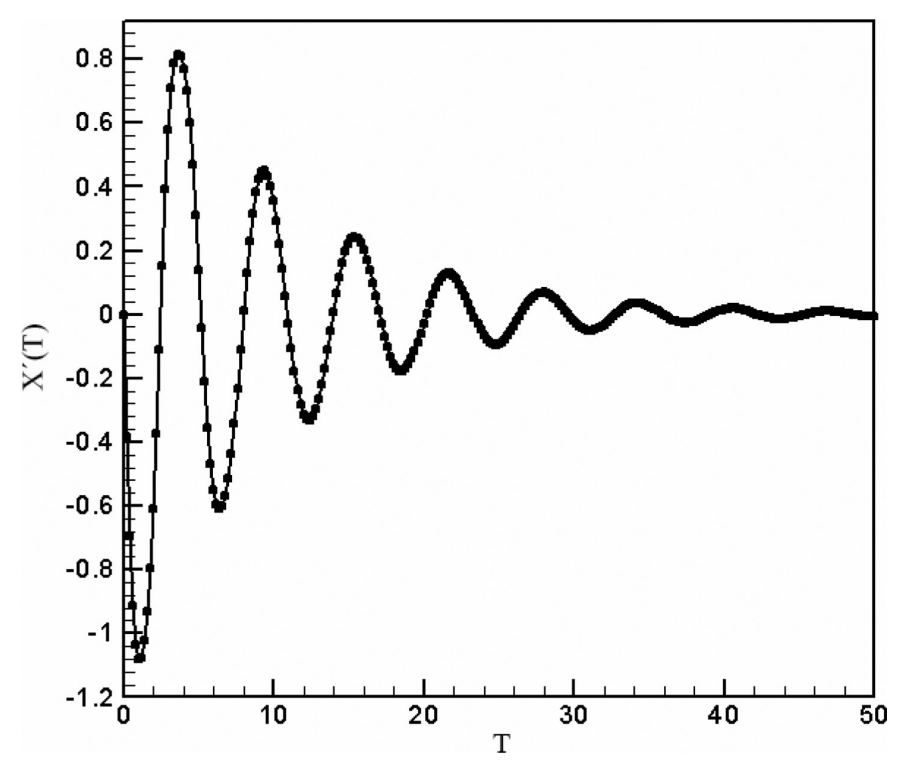

Fig. 5. The comparison between the numerical solution and DTM solution of $X^{\prime}(T)$ when $\zeta=0.1, \frac{K_{3}}{K_{1}}=1, \Omega=0.75$ and $P=0$ which solid line denote DTM solution and symbols show the numeric solution.

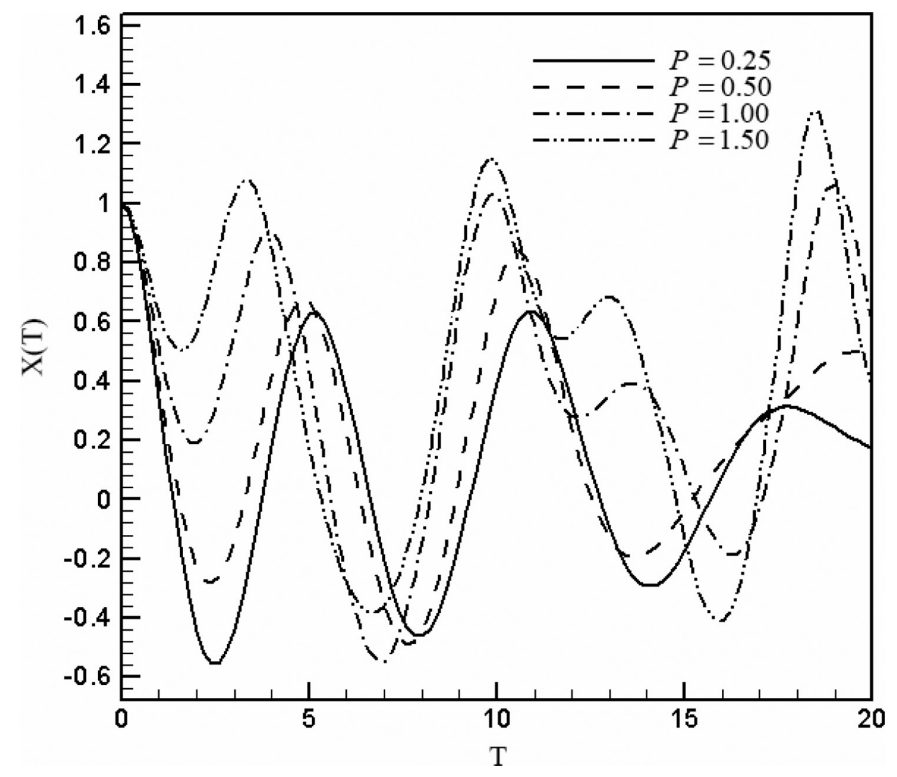

Fig. 6. The solution of $X(T)$ by DTM for various $P$, when $\zeta=0.1, \frac{K_{3}}{K_{1}}=1$ and $\Omega=0.75$. 


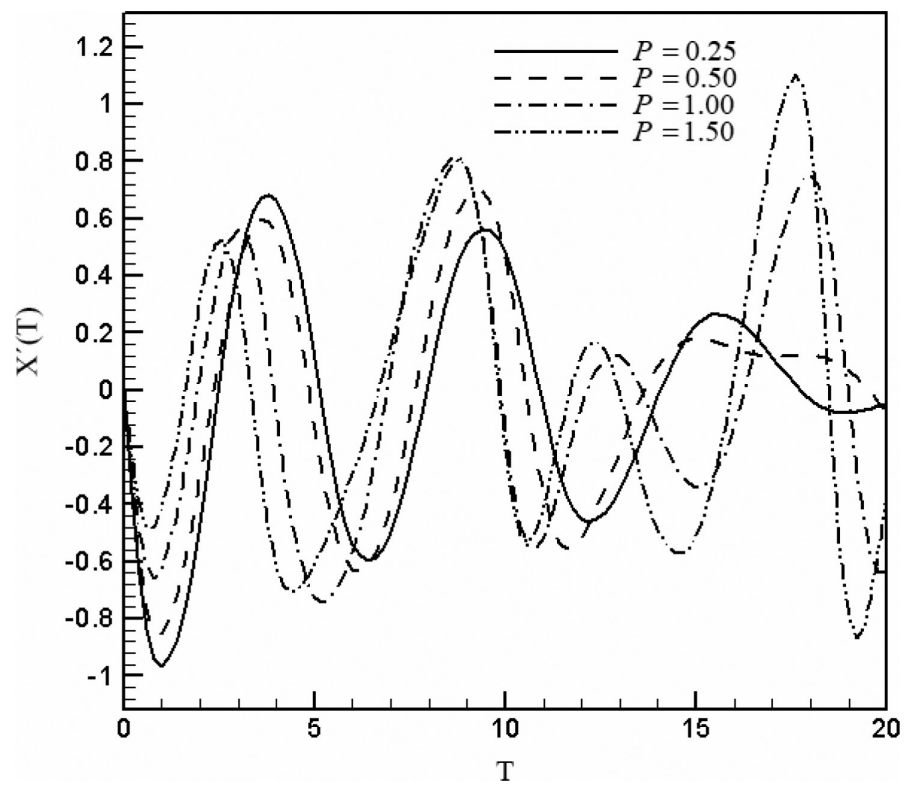

Fig. 7. The solution of $X^{\prime}(T)$ by DTM for various $P$, when $\zeta=0.1, \frac{K_{3}}{K_{1}}=1$ and $\Omega=0.75$.

DTM in terms of various frequency and damping ratios for $X(T)$ and $X^{\prime}(T)$ when $T=1$. Such results can be observed in Figs 4 and 5 considering the situation that there is no wind and then the damping would be decreased. As a result, good and clear agreements can be observed. Clearly, Figs 6 and 7 show the effect of normalized forcing amplitude on the $X(T)$ and $X^{\prime}(T)$ respectively. The main feature of the proposed analytical approach is that the solution is acceptable for all values of the constant parameters.

\section{Conclusion}

This paper has presented a dynamic model targeted at simulating the mechanical reaction of trees regarding a turbulent wind. Apparently, the presented dynamic models could help engineers calculate resonant effects of trees at all wind speeds. A differential equation derived from such manner has been solved successfully applying Differential Transformation Method (DTM) to predict the effects of loads on trees. The performance of the method was compared with the numerical one in order to introduce one of the most suitable methods for the prediction of tree behaviors. Applying this method transforms differential equations into algebraic equations in the $k$ domain. As a result, accurate results can be achieved easily. Furthermore, comparisons were made between DTM and Runge-Kutta (RK) illustrated that DTM facilitates the computational work in order to solve nonlinear problems that arise frequently to express nonlinear phenomenon especially in the field of biology, and consequently gives the solution rapidly if it compares with Runge-Kutta. Generally speaking, the work emphasized the method is a reliable technique to handle linear and nonlinear differential equations without using linearization, perturbation or restrictive assumptions.

\section{References}

[1] E.M. Everham, III., A comparison of methods for quantifying catastrophic wind damage to forests, in: Wind and Trees, M.P. Coutts and J. Grace, eds, Cambridge University Press, Cambridge, 1995, pp. 340-357.

[2] J.L. Haymond, D.D. Hook and W.R. Harms, Hurricane Hugo: South Carolina forest land research and management related to the storm. USDA Forest Service Southern Research Station Gen tech Rep SRS-5, 1996.

[3] H. Wandeler and R. Günter, Sturmschäden 1990: Lagebeurteilung aus der Sicht der eidgenössischen Forstdirektion, Schweiz Z Forstwes 142 (1991), 453-462. 
[4] C. Mattheck and H. Breloer, The Body Language Of Trees. HMSO, London, UK, 1994.

[5] C.J. Wood, Understanding wind forces on trees, 133-164. in: Wind and Trees. Cambridge University Press, M.P. Coutts and J. Grace, eds, Cambridge, UK, 1995.

[6] K. James, Dynamic loading of trees, Journal of Arboriculture 29(3) (2003), 165-171.

[7] J.J. Finnigan, Turbulence in plant canopies, Annu Rev Fluid Mech 32 (2000), 519-571.

[8] M. Denny, B. Gaylord, B. Helmuth and T. Daniel, The menace of momentum: dynamic forces on flexible organisms, Limnol Oceanogr 43 1998, 955-968.

[9] A.H. Nayfeh, Perturbation Methods, Wiley, New York, 2000.

[10] D.D. Ganji and A. Sadighi, Application of He's Homotopy-perturbation Method to Nonlinear coupled Systems of Reaction-diffusion Equations, International Journal of Nonlinear Sciences and Numerical Simulation 7 (2006), 411-418.

[11] S.S. Ganji, A. Barari, M. Najafi and G. Domairry, Analytical Evaluation of Jamming Transition Problem, Canadian Journal of Physics (2011), 729-738, Doi: 10.1139/p11-049.

[12] S.S. Ganji, D.D. Ganji, S. Karimpour and H. Babazadeh, Applications of a Modified He's Homotopy Perturbation Method to obtain secondorder approximations of the Coupled Two-Degree-Of-Freedom Systems, International Journal of Nonlinear Sciences and Numerical Simulation 10(3) (2009), 303-312.

[13] S.R. Seyed Alizadeh, G.G. Domairry and S. Karimpour, An approximation of the analytical solution of the linear and nonlinear integrodifferential equations by homotopy perturbation method, Acta Applicandae Mathematicae 104(3) (2008), 355-366.

[14] S.J. Liao, Beyond Perturbation: Introduction to Homotopy Analysis method, Chapman and hall/CRC, Boca Raton, 2003.

[15] Z. Ziabakhsh, G. Domairry and H.R. Ghazizadeh, Analytical solution of the stagnation-point flow in a porous medium by using the homotopy analysis method, Journal of the Taiwan Institute of Chemical Engineers 40(1) (2009), 91-97.

[16] J.H. He, Variational iteration method - a kind of non-linear analytical technique: some examples, International Journal of Non-Linear Mechanics 34(4) (1999), 699-708.

[17] S.S. Ganji, S. Karimpour and D.D. Ganji, He's Energy Balance and He's Variational Methods for Nonlinear Oscillations in Engineering, International Journal of Modern Physics B 23(3) (2009), 461-471.

[18] S.S. Ganji, M.G. Sfahani, S.M. Modares Tonekaboni, A.K. Moosavi and D. Domiri Ganji, Higher-order solutions of coupled systems using the parameter expansion method, Mathematical Problems in Engineering 23(32) (2009), 5915-5927.

[19] S.S. Ganji, A. Barari and D.D. Ganji, Approximate Analyses of Two Mass-Spring Systems and Buckling of a Column, Computers \& Mathematics with Applications 61(4) (2011), 1088-1095.

[20] S.S. Ganji, D.D. Ganji, M.G. Sfahani and S. Karimpour, Application of AFF and HPM to the Systems of Strongly Nonlinear Oscillation, Current Applied Physics 10(5) (2010), 1317-1325.

[21] S.S. Chen and C.K. Chen, Application of the differential transformation method to the free vibrations of strongly non-linear oscillators, Nonlinear Analysis: Real World Applications 10 (2009), 881-888.

[22] S.S. Ganji, A. Barari, G. Domairry and L.B. Ibsen, Differential Transform Method for Mathematical Modeling of Jamming Transition Problem in Traffic Congestion Flow, Central European Journal of Operations Research, in press.

[23] A.A. Joneidi, D.D. Ganji and M. Babaelahi, Differential Transformation Method to determine fin efficiency of convective straight fins with temperature dependent thermal conductivity, International Communications in Heat and Mass Transfer 36(7) (2009), 757-762.

[24] L.A. Miller, Structural dynamics and resonance in plants with nonlinear stiffness, Journal of Theoretical Biology 234 (2005), $511-524$.

[25] T. Kerzenmacher and B. Gardiner, A mathematical model to describe the dynamic response of a spruce tree to the wind, Trees 12 (1998), 385-394.

[26] D. Sellier, Y. Brunet and T. Fourcaud, A numerical model of tree aerodynamic response to a turbulent airflow, Forestry 81(3) (2008), 279-297.

[27] A. Fereidoon, M. Ghadimi, A. Barari, H.D. Kaliji and G. Domairry, Nonlinear Vibration of Oscillation Systems Using Frequency-Amplitude Formulation, Shock and Vibration (2011), DOI: 10.3233/SAV-2011-0633.

[28] A. Kimiaeifar, E. Lund, O.T. Thomsen and A. Barari, On Approximate Analytical Solutions of Nonlinear Vibrations of Inextensible Beams using Parameter-Expansion Method, International Journal of Nonlinear Sciences and Numerical Simulation 11(9) (2011), 743-753. 

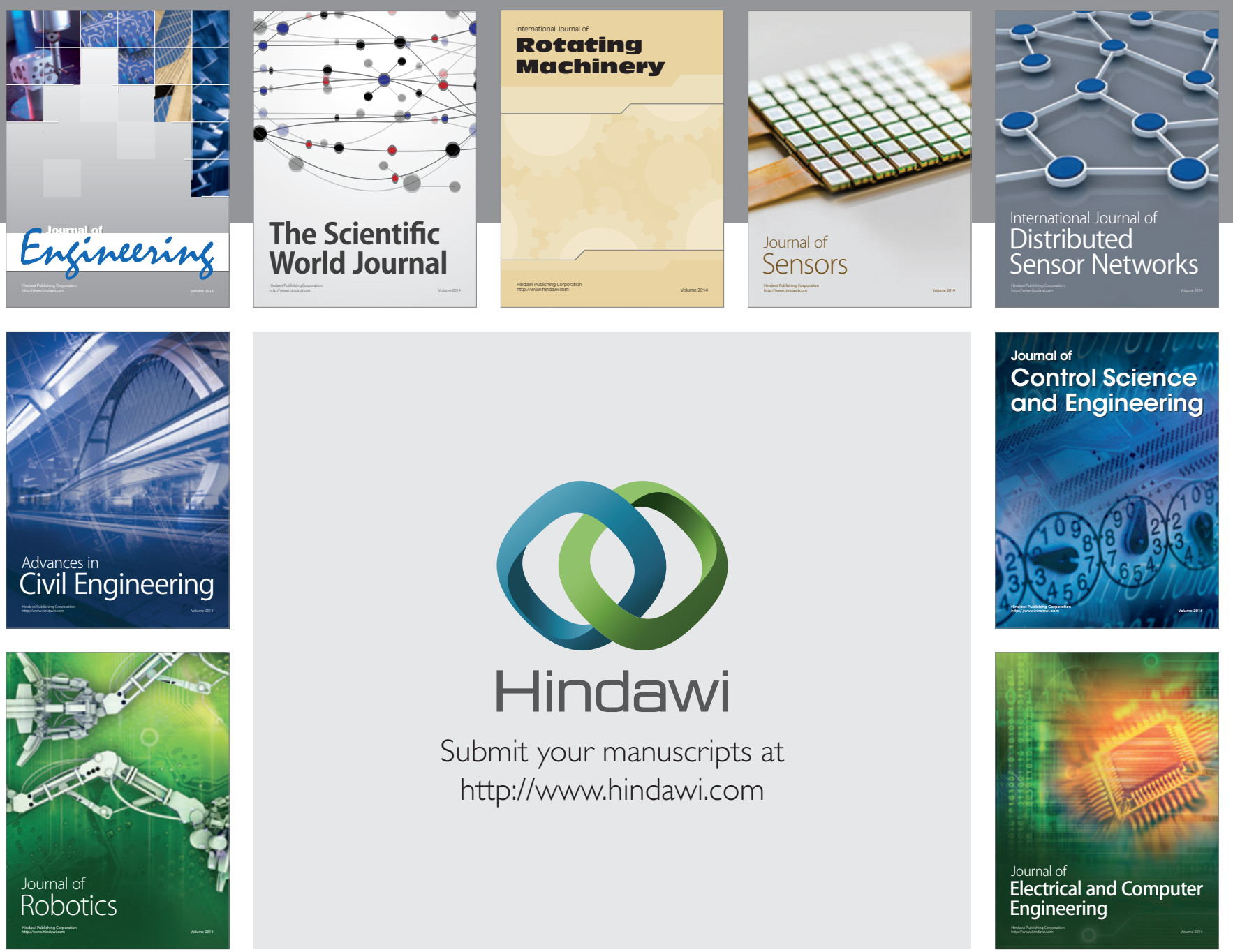

Submit your manuscripts at

http://www.hindawi.com
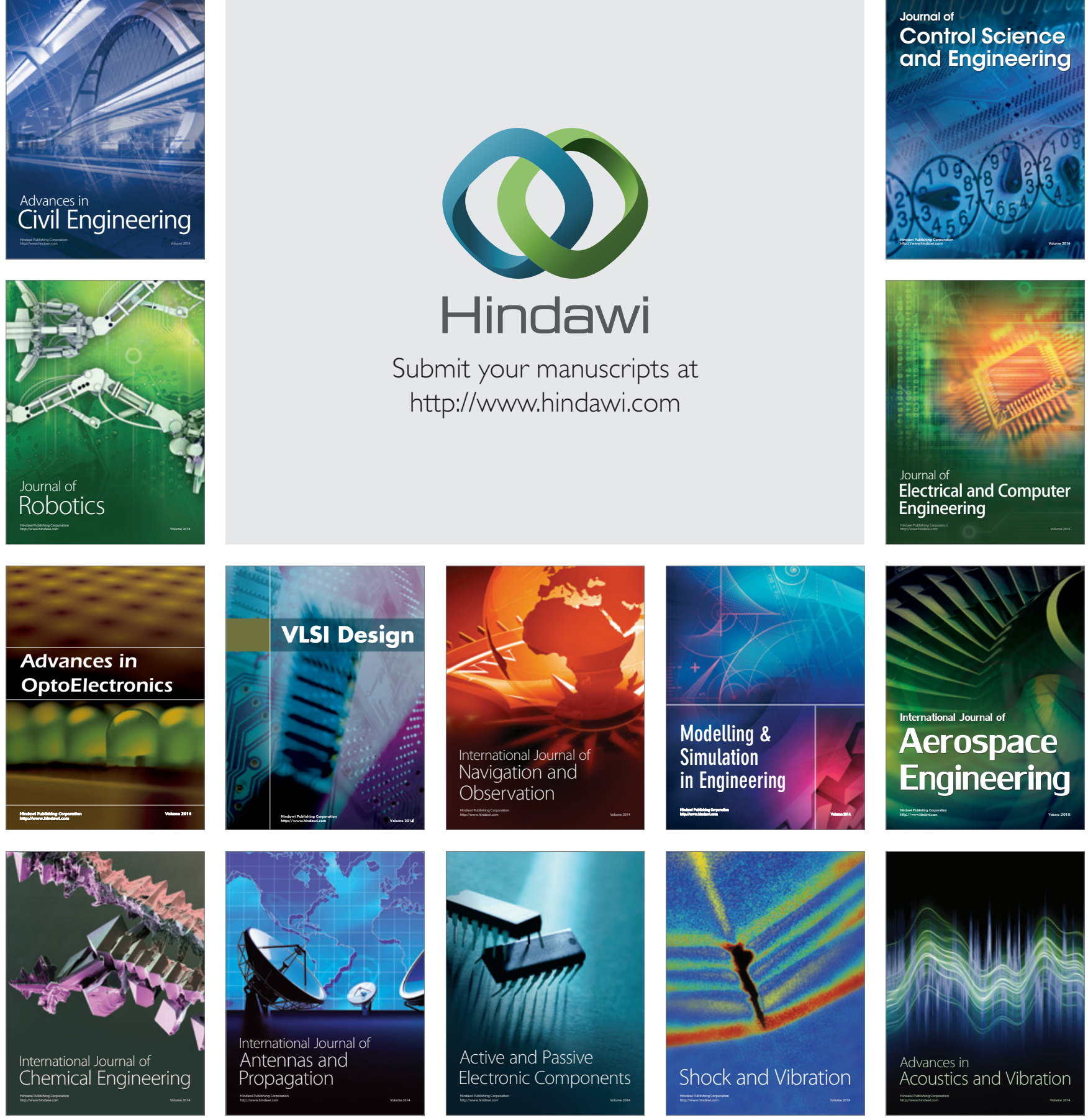